ORIGINAL PAPER

\title{
Assessment of Diagnostic VAlue of CYTOLOGiCAL EXAMINATION IN LUNG CARCINOMA IN OWN MATERIAL OBTAINED BY EBUS AND EUS METHODS
}

\author{
Piotr Wiśniewski ${ }^{1}$, Maciej GŁogowski ${ }^{2}$, WŁodzimierz Olszewski ${ }^{1}$
}

${ }^{1}$ Division of Pathology and Laboratory Diagnostics, Centre of Oncology - Institute in Warsaw, Poland

${ }^{2}$ Department of Lung and Chest Tumours, Centre of Oncology - Institute in Warsaw, Poland

\begin{abstract}
The aim of the study was the evaluation of the efficiency of cytological examination of the material obtained by endobronchial ultrasound-guided transbronchial needle aspiration (EBUS-TBNA) and endoscopic ultrasound bronchoscope-guided fine needle aspiration (EUSB-FNA) methods in the diagnosis of lung carcinoma. The usefulness was also assessed of the material obtained in that way for immunocytochemical and molecular tests in the diagnosis of non-small cell lung carcinoma. The material included cytological preparations obtained by EBUS and EUS methods. It was demonstrated that the technique made it possible to obtain diagnostic material from $94 \%$ of patients. A retrospective thorough analysis of those cases was the basis for the discussion of diagnostic difficulties.
\end{abstract}

Key words: lung carcinoma, EBUS-TBNA, EUSB-FNA, cytopathology.

\section{Introduction}

According to the National Registry of Tumours, in 2013 in Poland, 21,524 new cases of lung carcinoma were diagnosed; the tumour was registered in 14,609 men and 6915 women [1]. In view of anatomical-topographic conditions, the diagnosis of lung carcinoma is very complex; therefore, obtaining of tissue material, establishing the diagnosis, and assessment of the degree of tumour progression require invasive procedures. In recent years minimally-invasive examinations have been introduced to the clinical practice, including EBUS-TBNA (endobronchial ultrasound-guided transbronchial needle aspiration) and EUSB-FNA (endoscopic ultrasound-guided fine needle aspiration). These methods enable cytological material to be obtained by means of transbronchial biopsy from the tumour and, what is particularly important for the assessment of the progression stage of non-small-cell lung carcinoma (NSCLC), also from the mediastinal lymph nodes. Diagnostic efficiency is an important question, which, according to current requirements, is not only limited to making the diagnosis of a tumour but also should provide predictive data, important for planning the therapeutic process, such as the subtype of non-small-cell carcinoma and analysis of genetic mutations.

\section{Material and methods}

The cytological material obtained by EBUS-TBNA and EUSB-FNA methods in the Department of Lung and Chest Tumours, Maria Skłodowska-Curie Centre of Oncology - Institute (CO-I) in Warsaw from 1 January 2013 to 31 December 2014 were subjected to retrospective analysis. The examinations were carried out as part of routine diagnostic procedures in patients with: 1) lung tumour, not verified morphologically; 2) mediastinal lymph node enlargement of undetermined character; 3) suspicion of a recurrence after surgery for NSCLC; 4) diagnosed NSCLC, in order to assess the lymph node 
status and possibly to qualify the patients for a surgical procedure. A total of 324 patients were examined by the EBUS-TBNA and EUSB-FNA methods in the CO-I, taking 710 aspirates by the EBUS-TBNA and 18 by the EUSB-FNA method. The examinations were carried out in 146 women and 178 men. The preparations were initially examined by nine pathologists. The microscopic preparations (2970 smears in total), which had been archived in the Division of Pathology and Laboratory Diagnostics, Centre of Oncology - Institute in Warsaw, were subjected to retrospective consultation assessment. In 25 cases lack of at least one preparation was found, and these preparations were sent to the Laboratory of Molecular Tests, DPLD, CO-I, which was taken into account during assessment of the diagnostic value and of the usefulness for molecular tests. Two consultants in pathology performed the simultaneous evaluation assessment. Apart from establishing the microscopic diagnosis and qualification for immunocytochemical tests, also the usefulness of the cytological material for molecular tests, and diagnostic difficulties that occurred in the examined cytological smears, were determined.

The endoscopic examinations, both EBUS-TBNA and EUSB-FNA, were performed with an EVIS EXERA II (OLYMPUS BF-UC180F) ultrasound video bronchofibrescope. The aspirates were taken from the mediastinal lymph nodes, pulmonary hilar lymph nodes, and interlobar lymph nodes. The obtained aspirates were fixed in $95 \%$ alcohol and all smears were routinely stained with haematoxylin and eosin (HE).

The aspirate was assumed as diagnostic positive if malignant cells were found in the smears. The criteria of assessment of tumour cell atypia were accepted according to commonly known criteria used in the cytological diagnostics [2]. Positive diagnoses (i.e. diagnoses of malignant tumour) included non-small cell lung carcinoma, small-cell lung carcinoma, carcinoma cells (type indefinable), and malignant cells. The conditions of making the diagnosis of a malignant tumour included the presence of numerous dispersed malignant cells or at least five groups formed of several malignant cells. As diagnostic negative cases, such preparations were assumed, in which only lymph node structure was found, including lymphocytes and macrophages, without the presence of malignant cells.

As non-diagnostic, these preparations were regarded, in which no malignant cells were present and no diagnostic counts of lymph node cells were found. Simultaneously, as a criterion of diagnostic positivity, at least one smear was accepted containing numerous lymphocytes and/or macrophages with pneumoconiotic changes. In doubtful cases at least five fields of vision in cell-rich areas were assessed at object glass magnification $40 \times$ and, as non-diagnostic, these cases were accepted, in which in the cell-rich fields less than 40 lymphocytes / 1 field of vision were present (40 $\times$ objective lens power).

A diagnostic result of even one group of lymph nodes with the presence of malignant cells was treated as a positive case, irrespective of whether the aspirates of other groups of lymph nodes in that patient were negative or non-diagnostic. Similarly, each case in which in at least one group of diagnostic lymph node cells were found was treated as a diagnostic negative result, on condition that in other groups of lymph nodes no malignant cells were seen. Cases were regarded as non-diagnostic if in all smears done in the patient, irrespective of their number, no diagnostic material was found. It should be mentioned that apart from the absence of malignant cells or the presence of a diagnostic count of lymph node cells, other reasons for acceptance of the smears as nondiagnostic included non-optimal fixation of the cells or their damage, e.g. as a result of crushing at the time of making the smears.

\section{Results}

The EBUS-TBNA and EUSB-FNA examinations were carried out by three bronchoscopy specialists, described as A, B, and C, who performed 16, 167, and 175 procedures, respectively.

The analysis of non-diagnostic, diagnostic negative, and positive results obtained by individual bronchoscopists demonstrated no statistically significant differences ( $\chi^{2}$ test, $\mathrm{p}=0.6158$ ), which was evidence of the repeatability of the method irrespective of individual skills of the doctor performing the procedure, and of the fact that the procedures were only performed by the doctors, who had acquired adequate skills during the training courses.

Most frequently, the material was taken from two groups of lymph nodes - 159 cases, while examination of only one group of lymph nodes was done in 102 cases, and the biopsy of three lymph node groups was carried out in 92 patients. The biopsy of four locations was done in seven cases, and in one patient the biopsy was taken from five locations (Table I).

In 33 cases the examination was repeated, most frequently due to absence of diagnostic material. For that reason, the procedure was repeated in $30 \mathrm{pa}-$ tients. Two patients had a repeated biopsy in order to obtain material that could be qualified for molecular tests. In one case of diagnosed tumour metastasis to the N1 group of lymph nodes a repeated examination was performed to rule out or confirm a metastasis in a lymph node from the N2 group.

In four patients the repeated procedure was done two times because the first biopsy failed to provide diagnostic material. As a result of the repeated 
Table I. Number of biopsies performed during single EBUS and EUS examination

\begin{tabular}{lc}
\hline Number OF SIMULTANEOUS BIOPSIES & NumBER OF CASES \\
\hline One location & 104 \\
\hline Two locations & 159 \\
\hline Three locations & 92 \\
\hline Four locations & 7 \\
\hline Five locations & 1 \\
\hline
\end{tabular}

biopsies, in 19 out of 30 cases positive results were obtained. In two patients the obtained repeated cytological material was qualified for molecular tests. On the other hand, in one patient, based on the repeated examination, the lymph node status was changed from N1 to N2. In 11 patients in the repeated biopsies only the cellular elements of lymph node structure were found.

In 20 patients no diagnostic material was obtained and no repeated biopsy by the EBUS-TBNA/ EUSB-FNA method was performed.

Significant differences were observed in the frequency of performing biopsies of various groups of lymph nodes. Most frequently, biopsies of lymph node group 4 were done, the second in the frequency of the biopsies was group 7, and least frequently biopsies were taken from groups 6, 8, and 5 .

The greatest number of diagnostic aspirates were also obtained in group 4, while most non-diagnostic results were found in group 7 (Fig. 1).

Taking into account the proportions of the examinations performed, the highest percentage of diagnostic results was also obtained in group 4, while the highest percentage of non-diagnostic results was found in group 7 . Jointly, the non-diagnostic biopsies accounted for $20 \%$ of all examinations.

In the cytological assessment of 728 examinations in 324 patients, malignant cells were found in 198 patients (61.1\%). In 106 patients $(32.7 \%)$ no malignant cells were found, only cellular elements of lymph node structure were seen. The material was assessed as non-diagnostic in 20 patients (6.2\%).

The positive cases, in which malignant cells were found, were divided into the following groups: 1) small cell carcinoma; 2) squamous cell carcinoma; 3) adenocarcinoma; 4) non-small cell lung carcinoma not otherwise specified (NSCLC NOS) (no possibility of type determination in microscopic examination); and 5) tumour cells (this group included non-epithelial primary lung tumours and malignant metastases to the lungs) (Table II).

Malignant tumours were detected in 349 out of 728 biopsies aspirates.

In 106 examinations only cellular elements of lymph node structure were found: lymphocytes and

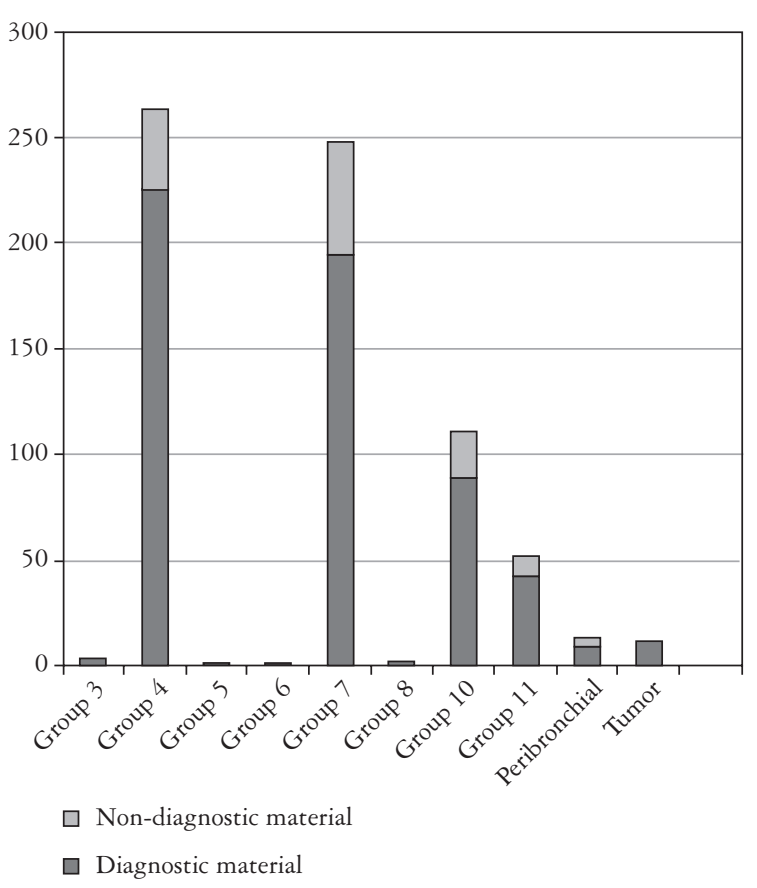

Fig. 1. Number of diagnostic and non-diagnostic aspirates in groups of lymph nodes and in other locations

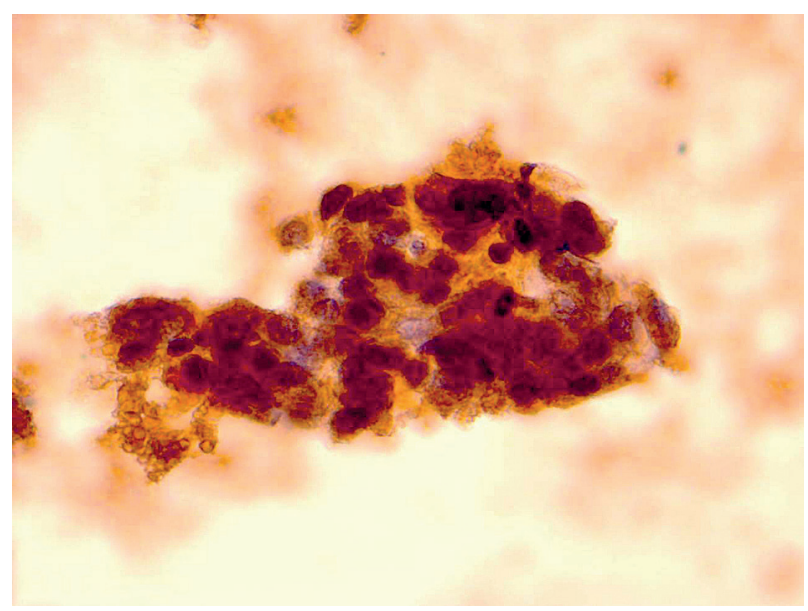

Fig. 2. Nuclear TTF-1 positivity in adenocarcinoma cells

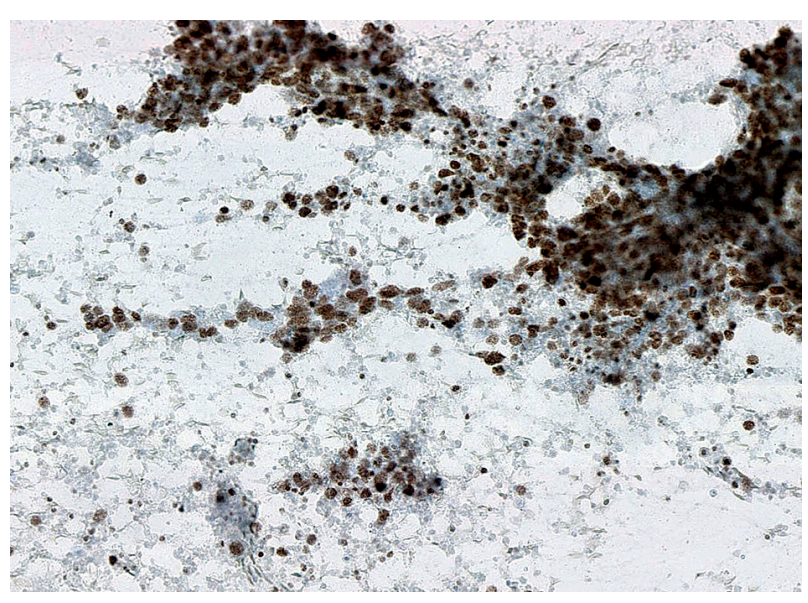

Fig. 3. Immunoexpression of p40 in squamous cell carcinoma 
Table II. Number of patients with the diagnosis of a tumour including its type, non-diagnostic result, and diagnostic negative result

\begin{tabular}{lc}
\hline Small-cell carcinoma & 41 \\
\hline Squamous cell carcinoma & 57 \\
\hline Adenocarcinoma & 58 \\
\hline Non-small-cell carcinoma & 33 \\
\hline Tumour cells & 9 \\
\hline Lymphonodular structure cells & 106 \\
\hline Non-diagnostic material & 20 \\
\hline
\end{tabular}

histiocytes, the latter ones frequently contained pneumoconiotic material.

Immunocytochemical stains were performed only in 13 cases. A specific reaction was observed and usefulness for the determination of the type of nonsmall-cell carcinoma (Fig. 2, Fig. 3) was found. Also, confirmation of metastasis of large bowel cancer, seminoma, and prostatic cancer was obtained. Immunohistochemical tests were only done in doubtful cases, without unequivocal signs of glandular or planoepithelial differentiation, preserving the diagnostic material for predictive testing.

An assessment of EGFR gene status was done in 32 patients out of 91 with diagnosed adenocarcinoma or non-small cell not otherwise specified (NOS) carcinoma. In five cases an examination was ordered of the available histological material: in two cases of the material from cell-blocks and in the remaining 25 cases, from cytological preparations. The cytological material made it possible to obtain diagnostic results of the molecular test. Only in one patient was the presence of EGFR gene mutation found; the test was done on cytological material. In two cases, in view of absence of the material, which could have been qualified for molecular testing in the patients with diagnosed lung adenocarcinoma, a repeated EBUS-FNA biopsy was carried out in order to obtain cytological smears, which could have been qualified for the assessment of EGFR gene status. In the remaining 56 cases of adenocarcinoma and 33 cases of non-small-cell carcinoma without signs of squamous epithelial differentiation, at least one of the smears obtained by the EBUS-TBNA or EUSB-FNA method was qualified for molecular tests. The usefulness of the material obtained by the EBUS and EUS method for molecular tests has been well documented in the literature $[3,4,5,6,7,8,9,10]$.

\section{Discussion}

The analysis of the retrospectively assessed cases made it possible to call attention to the difficulties encountered by cytopathologists reagrding the cytological material.

One of the most frequent problems is the difficulty in distinguishing squamous cell carcinoma from metaplastic cells of the squamous epithelium, often seen in the bioptates of lymph nodes carried from the respiratory tract during biopsies. It results from the absence of an evident cytological atypia of the cells of highly-differentiated squamous cell carcinoma. In such cases immunohistochemical stains only allow confirmation of a squamous epithelial differentiation but without the possibility to demonstrate the neoplastic character of the cells. The presence of necrosis and mitotic activity in tumour cells is of help in distinguishing metaplasia from cancer. Also, the bronchial epithelial cells, particularly the reserve cells, can show signs of reactive atypia. Of key importance in distinguishing reactive changes from cancer is finding the intermediate stages, from normal cells to more and more atypical cells; such stages are present in the reactive changes. In the case of cancer, we usually encounter two populations of cells: normal and carcinomatous. Even more difficult is distinguishing, in cytological smears, lymph node structure cells from non-Hodgkin lymphoma cells; the latter are usually monomorphic and of similar size and stainability of cell nuclei. Lymph node lymphocytes are characterised by diversity, with predominance of mature lymphocytes, which are accompanied, among other cells, by histiocytes and plasmocytes.

Poorly differentiated tumours lose the cytological features typical of the tissues they originate from, which renders the diagnosis of cancer type difficult. In order to establish the direction of differentiation it may be necessary to use immunocytochemical methods, although in that situation it should be stressed that it is the immunocytochemical profile that corresponds to the given type of cancer $[11,12,13,14]$.

Carcinoma of the lung is the most frequently developing primary malignancy of that organ. It should, however, be kept in mind that the lung is a very frequent location of metastases from other organs. In order to avoid making a mistake that is very unfavourable for the patient, in the assessment of the primary and metastatic tumour, the clinical data from possible oncological history of the patient should always be learned. It may be indispensable to perform differentiating immunocytochemical stains. In the case of squamous cell carcinoma, even on the basis of the immunoprofile, it cannot be established by cytological evaluation whether the tumour is primary or metastatic. The detection of HPV infection may be helpful, which is typical of uterine cervical cancer and cancer of the head and neck, while lung carcinoma is usually hrHPV negative [15]. Intestinal adenocarcinoma is characterised by a high morphological similarity to primary mucus-producing lung carcinoma 
of TTF1-negative, CK20-positive immunoprofile; therefore, the basic immunoprofile may not give a decisive result [16]. Besides that, in primary lung adenocarcinoma of intestinal type, a positive expression is possible of the antibody typical of gastrointestinal adenocarcinoma - CDX2. In such situations, the clinical data including information on the oncological history of the patient are of key importance.

The analysis of the false negative cases demonstrates the role of the quality of smears in establishing the correct diagnosis. Non-optimal fixation, drying, or mechanical damage cause the cells (both normal and malignant) to become difficult to assess in cytological examination - the morphological details of the cell nucleus are blurred and the nucleus/cytoplasm ratio is also disturbed. For that reason, the certainty is frequently lacking as to whether the malignant cells are changed to such a degree, or the atypia is only apparent. In such situations the best preserved diagnostic cells should always be sought in the preparation. If such cells are absent, then, in spite of the presence of suspected cells, the preparation should be regarded as non-diagnostic. That will help avoid false positive results. In the reports by the authors of many papers false positive results are extremely rare, accounting for less than $3 \%$ of the diagnoses [17].

There is no commonly accepted criterion making it possible to confirm with certainty that an aspirate comes from a lymph node. If among the cells very numerous lymphocytes are present, among which small mature lymphoid cells predominate, accompanied by larger cells of lymphatic follicles and histiocytes, it can be said with a high probability that the aspirate comes from a lymph node. Unfortunately, such smears were very few in the analysed material; usually lymphocytic cells were mixed in various proportions with blood corpuscular elements. In such a situation clusters of lymphocytes should be looked for, and in those regions at least five large fields of vision should be found $(40 \times$ objective, $400 \times$ magnification), in which more than 40 lymphocytes are present. However, it is an arbitrary criterion, and it is not commonly recognised $[18,19]$. Other criteria have been recommended by Patelli [18], according to whom, a preparation should be regarded as lymph node smear if lymphocytes account for more than $30 \%$ of all cells in the smear. On the other hand, Nayak [19] suggests that an aspirate comes from a lymph node if in more than five fields of vision $(10 \times$ objective; $100 \times$ magnification $)$ more than 100 lymphocytes/one field of vision are present. All those criteria assume that lymphocytes are always present in the lymph nodes, but it is known that lymph nodes undergo fibrosis, hyalinisation, or pneumoconiotic changes. In such nodes it is difficult to find preserved lymphocytes, and then the presence of histiocytes containing pneumoconiotic material may be helpful. It should be kept in mind, however, that such cells may be present, e.g., in pulmonary alveoli. In a situation when the lymphonodular origin of the aspirate cannot be unequivocally confirmed, that information should be included in the cytological diagnosis. We used in our practice a combination of criteria according to Kramer (at least one smear with an area of small lymphocytes or dendritic cells) [20], and in doubtful cases we used the Alsharif criteria (more than 40 lymphocytes in a large field of vision in the most cell-rich areas) [21]. The requirements of modern oncology impose the necessity of determination of non-small cell cancer subtype, which is difficult and sometimes even impossible in cytological examination. A limitation of the EBUS and EUS methods, similarly as in the case of cytological material obtained in other ways, is the lack of the possibility of determination of histoformative structures, which are helpful in histological assessment. Another important feature of the tumour, which cannot be assessed in cytology, is the spread of the tumour through the alveolar space (STAS) [21]. Negative and non-diagnostic results are an important problem in EBUS-TBNA and EUSB-FNA examinations. In various centres the percentage of non-diagnostic material obtained by the EBUS-TBNA method ranges from $5 \%$ to $12.5 \%[22,23,24,25]$. It should be mentioned that the percentage of non-diagnostic materials could have been influenced by various criteria of smear diagnosticity and application (or not) of rapid on-site evaluation and cell-block.

Negative results require a clinical-radiological correlation and a possible histological verification whether they are true negative, or false negative. On the other hand, non-diagnostic cases always require verification in another cytological or histological examination. It should be stressed that lack of histological verification is a limitation of the analysis discussed, which results from the specificity of the oncological centre, which most frequently deals with patients with advanced disease, usually at an inoperable stage. Fifty-two patients in the study group had histopathological diagnoses, but only in 10 of them the lymph nodes were verified by histological examination, while in the remaining cases the histological examinations concerned bronchial oligobioptates and tumours of extrapulmonary origin. In the examination of nine lymph nodes assessed as negative based on EBUS/EUS method, absence of tumour structure was confirmed, and in one case assessed cytologically as positive, a carcinomatous metastasis to the lymph node was confirmed. In view of the very low number of histological examinations of lymph nodes previously examined by EBUS/EUS method, no statistical analysis of those cases has been conducted. EBUS-TBNA and EUS-FNA are methods recently introduced to routine diagnostics of lung 
tumours, which, as with any cytological examination, require close co-operation of the radiologist, clinician, and pathologist. The conditions of diagnostic success include adequate qualification of patients, indication of lymph node groups most suspected on radiography, skilful biopsy of the aspirate, and professional microscopic assessment of the smears. The role of the doctor taking the material for cytological examination includes acquiring of the maximal possible amount of diagnostic material indispensable for microscopic and immunocytochemical diagnostic procedures and, more and more frequently and to a greater and greater extent, for molecular diagnostics [26].

\section{Conclusions}

Based on two years of observations, we can say that cytological materials obtained by the EBUS-TBNA and EUSB-FNA methods are useful for the diagnosis of lung cancer, particularly for the assessment of the stage of primary non-small cell lung carcinoma.

The material obtained by EBUS-TBNA and EUSBFNA methods usually makes it possible to determine the histological type of the cancer, which sometimes requires application of immunohistochemical tests.

The cytological material obtained in that way can be qualified for molecular tests indispensable in modern diagnostics of non-small cell lung cancer and so-called targeted therapy. The results obtained demonstrate that EBUS-TBNA and EUSB-FNA are examinations providing data indispensable for making therapeutic decisions. The co-operation between an experienced bronchoscopy specialist and pathologist is necessary to obtain optimal results.

\section{The authors declare no conflict of interest.}

\section{References}

1. Krajowy Rejestr Nowotworów: http: //epid.coi.waw.pl/krn/.

2. Koss LG, Woyke S, Olszewski W. Aspiration biopsy. Cytologic interpretation and histologic bases. Igaku-Shoin. Second edition 1992; 288-321.

3. da Cunha Santos G, Saieg MA, et al. EGFR gene status in cytological samples of non small cell lung carcinoma: Controversies and opportunities. Cancer Cytopathol 2011; 119: 80-91.

4. Boldrini L, Gisfredi S, Ursino S, et al. Mutational analysis in cytological specimens of advanced lung adenocarcinoma: A sensitive method for molecular diagnosis. J Thorac Oncol 2007; 2: 1086-1090.

5. Cai G, Wong R, Chhieng D, et al. Identification of EGFR mutation, KRAS mutation, and ALK gene rearrangement in cytological specimens of primary and metastatic lung adenocarcinoma. Cancer Cytopathol 2013; 121: 500-507.

6. Li AR, Chitale D, Riely GJ, et al. EGFR Mutations in Lung Adenocarcinomas. Journal of Molecular Diagnostics 2008; 10 , 3: $242-248$

7. Izumo T, Matsumoto Y, Chavez C, et al. Re-biopsy by endobronchial ultrasound procedures for mutation analysis of non- small lung cancer after EGFR thyrosine kinase inhibitor treatment. Pulm Med 2016; 16: 106.

8. Billah S, Stewart J, Staerkel G, et al. EGFR and KRAS mutations in lung carcinoma: Molecular testing by using cytology specimens. Cancer Cytopathol 2011; 119: 111-117.

9. Khode R, Larsen DA, Culbreath BC, et al. Comparative study of epidermal growth factor receptor mutation analysis on cytology smears and surgical pathology specimens from primary and metastatic lung carcinomas. CancerCytopathol 2013; 121 : 361-369.

10. Li AR, Chitale D, Riely GJ, et al. EGFR mutations in lung adenocarcinomas: clinical testing experience and relationship to EGFRgene copy number and immunohistochemical expression. J Mol Diagn 2008; 10: 242-248.

11. Travis WD, Brambilla E, Burke AP, et al. Introduction to The 2015 World Health Organization Classification of Tumors of the Lung, Pleura, Thymus, and Heart. J Thorac Oncol 2015; 10: 1240-1242.

12. Langfort R. Klasyfikacja pTNM raka płuca. Pol J Pathol 2010; 61 (suppl 1).

13. Travis WD, Brambilla E, Noguchi M, et al. International Association for the Study of Lung Cancer/American Thoracic Society/European Respiratory Society International Multidisciplinary Classification of Lung Adenocarcinoma. J Thorac Oncol 2011; 6: 244-285.

14. Langfort R, Szołkowska M, Szczepulska-Wójcik E, et al. Zalecenia dotyczące oceny mikroskopowej małych wycinków i rozmazów cytologicznych w diagnostyce raka niedrobnokomórkowego płuca na podstawie rekomendacji przedstawionych przez IASLC/ATS/ERS. Pneumonol Alergol Pol 2011; 80: 172-177.

15. van Boerdonk RA, Daniels JM, Bloemena E, et al, High-risk human papillomavirus-positive lung cancer: molecular evidence for a pattern of pulmonary metastasis. J Thorac Oncol 2013; 8: 711-718.

16. Dabbs D. Diagnostic immunohistochemistry. 2nd edition. Churchill Livingstone Elsevier, Philadelphia 2006.

17. Junhong Jiang, Browning R, Lechtzin N, et al. TBNA with and without EBUS: a comparative efficacy study for the diagnosis and staging of lung cancer. J Thorac Dis 2014; 6: 416420.

18. Patelli M, Lazzari A, Poletti V, et al. Role of fiberoscopic transbronchial needle aspiration in the staging of $\mathrm{N} 2$ disease due to non-small lung cancer. Ann Thorac Surg 2002; 73: 407-411.

19. Nayak A, Sugrue C, Koenig S, et al. Endobronchial ultrasound guided transbronchial needle aspirate (EBUS-TBNA): a proposal for on-site adequacy criteria. Diagn Cytopathol 2012; 40: 128-137.

20. Kramer H, Sanders J, Post WJ, et al. Analysis of cytological specimens from mediastinal lesions obtained by endoscopic ultrasound-guided fine-needle aspiration. Cancer 2006; 108: 206-211.

21. Alsharif M, Andrade RS, Groth SS, et al. Endobronchial ultrasound-guided transbronchial fine-needle aspiration: the University of Minnesota experience, with emphasis on usefulness, adequacy assessment, and diagnostic difficulties. Am J Clin Path 2008; 130: 434-443.

22. Young Rak Choi, Jin Young An, Mi Kyeong Kim, et al. The diagnostic efficacy and safety of endobronchial ultrasound-guided transbronchial needle aspiration as an initial diagnostic tool Korean J Intern Med 2013; 28: 660-667.

23. Herth FJF, Eberhardt R, Vilmann P, et al. Real-time endobronchial ultrasound guided transbronchial needle aspiration for sampling mediastinal lymph nodes. Thorax 2006; 61: 795798.

24. Navani N, Brown JM, Nankivell M, et al. Suitability of endobronchial ultrasound-guided transbronchial needle aspiration specimens for subtyping and genotyping of non-small cell lung cancer. Am J Respir Crit Care Med 2012; 185: 1316-1322. 
25. Feller-Kopman D, Yung RC, Burroughs F, et al. Cytology of endobronchial ultrasound-guided transbronchial needle aspiration: a retrospective study with histology correlation. Cancer Cytopathol 2009; 117: 482-490.

26. Langfort R. Rola badań patomorfologicznych w wyborze metody leczenia raka płuca. Onkologia po Dyplomie 2016; 2: 42-47.

\section{Address for correspondence}

Piotr Wiśniewski

Division of Pathology and Laboratory Diagnostics

Centre of Oncology - Institute

Roentgena 5

02-781 Warsaw, Poland

e-mail: piotr.patolog@gmail.com 